\title{
Nutrition for Health and Fitness: Sugar and Other Sweeteners ${ }^{1}$
}

\section{Linda Bobroff ${ }^{2}$}

\section{Why the concern?}

Most people enjoy the sweet taste of sugar. In fact, very young infants smile when a drop of sugar water is put on their lips! However, sugars provide calories, but none of the nutrients that we need for good health. People who eat too many foods that are high in added sugars often consume more calories and fewer nutrients than they need. This can lead to obesity and a variety of health problems. There is strong evidence that most Americans would be healthier if they consumed fewer foods and beverages that contain added sugars. Let's see why excessive intake of added sugars is causing health problems for many Americans:

- Sugar is a source of "empty" calories. Eating or drinking excess calories can contribute to obesity and associated health problems.

- Foods and beverages high in added sugars often replace healthier foods and drinks in the diet. This can result in a nutrient-poor diet that does not support good health.

- Sugar contributes to tooth decay, which is a major public health problem in the United States, especially among low-income families.

This publication provides information about sugars and other sweeteners in the American diet. It describes hidden sources of added sugars in foods to help those who want to reduce sugar intake find the added sugars in their diets. The section on high-intensity sweeteners looks at the characteristics of each approved sweetener.

\section{What is sugar?}

Sugar is one type of carbohydrate. Carbohydrate is one of the nutrients that provide calories in our diets. The other calorie-containing nutrients are protein and fat. There are various types of sugars, all of which provide about four calories per gram, the same as other carbohydrates and protein. All sugars are nutritive sweeteners, meaning that they are sweet and provide calories. Sweeteners that provide few or no calories and that we use as sugar substitutes are known as high-intensity sweeteners.

\section{Nutritive Sweeteners}

The most common nutritive sweetener is table sugar, or sucrose. Sucrose is a double sugar (disaccharide) made up of fructose and glucose, two simple sugars (monosaccharides). Fructose and glucose are found naturally in fruits and honey. Sucrose also is refined from sugar beets or sugarcane and is found in a wide variety of processed foods, some of which (e.g., salad dressings and ketchup) are not even sweet!

Fructose is sweeter than other sugars and is used to sweeten many foods. Because it is so sweet, a smaller amount can be used, which adds fewer calories. However, fructose does not taste sweeter than other sugars in all foods and beverages, and it is more expensive than sucrose.

1. This document is FSHN20-46, one of a series of the Food Science and Human Nutrition Department, UF/IFAS Extension. Original publication date October 2020. Visit the EDIS website at https://edis.ifas.ufl.edu for the currently supported version of this publication.

2. Linda Bobroff, professor emeritus, Department of Family, Youth and Community Sciences; UF/IFAS Extension, Gainesville, FL 32611.

The Institute of Food and Agricultural Sciences (IFAS) is an Equal Opportunity Institution authorized to provide research, educational information and other services

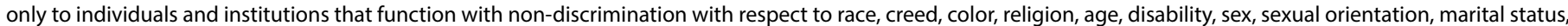

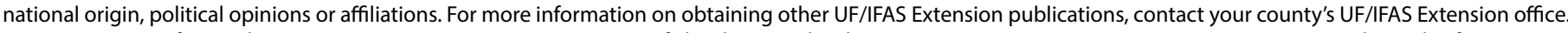
U.S. Department of Agriculture, UF/IFAS Extension Service, University of Florida, IFAS, Florida A \& M University Cooperative Extension Program, and Boards of County Commissioners Cooperating. Nick T. Place, dean for UF/IFAS Extension. 
Lactose, or milk sugar, is another disaccharide, containing the monosaccharides glucose and galactose. Lactose provides a small percentage of the sugar intake in the American diet. As you can tell from the taste of milk, lactose is not a very sweet sugar.

Another sugar found in a variety of foods is the disaccharide maltose, which consists of two glucose molecules. Maltose is used for brewing beer and is formed by yeast in bread making. Legumes (beans and lentils) and cereals contain small amounts of maltose.

Table 1. Common sugar types.

\begin{tabular}{|l|l|}
\hline Monosaccharides & \multicolumn{1}{c|}{ Disaccharides } \\
\hline Glucose (Dextrose) & Maltose $=$ Glucose + Glucose \\
\hline Fructose & Sucrose $=$ Glucose + Fructose \\
\hline Galactose & Lactose $=$ Glucose + Galactose \\
\hline
\end{tabular}

Table 2. Relative sweetness of sugars and other sweeteners (FDA 2015; Mahan \& Escott-Stump 2008).

\begin{tabular}{|l|}
\hline Advantame (sweetest) \\
\hline Neotame \\
\hline Sucralose \\
\hline Saccharin \\
\hline Stevia \\
\hline Aspartame and acesulfame-K \\
\hline Cyclamate (banned in the US) \\
\hline Fructose \\
\hline Luo Han Guo extract \\
\hline Sucrose and xylitol \\
\hline Glucose \\
\hline Sorbitol and mannitol \\
\hline Galactose and maltose \\
\hline Lactose (least sweet) \\
\hline
\end{tabular}

\section{Why We Eat Sugar}

Sugar, syrups, and other sweeteners are found in a variety of foods as added ingredients. Foods with added sugars include candy, pastries, pies, cakes, cookies, cereals, beverages, salad dressings, and many others. Health professionals are concerned about the added sugars in these types of foods and other "hidden" sources of sugars. Fruits, which contain naturally occurring sugars, are part of a healthful diet. They provide a variety of nutrients and fiber and are great choices for wholesome sweet desserts or snacks. Milk contains the natural sugar lactose, which is a concern only for those who are unable to digest this sugar. Lactose-free milk is an option for those who are lactose-intolerant, and yogurt is often well tolerated.
Added sugars play a versatile role in foods. They give foods sweetness, a quality that many people enjoy. Besides improving the flavor of foods, sugars add texture and color to baked goods and help to thicken, firm, or preserve such foods as puddings, jams, and jellies. Eating these foods in moderation is fine, but many people consume much more added sugar than is healthy.

\section{How Much Sugar We Eat}

In the United States, in 2003 to 2010, added sugars made up about $14 \%$ of calorie intake for people six years and older. This does not count natural forms of sugar in fruits and dairy products. Adults (but not children) with lower incomes tend to have higher intakes of added sugars, which may play a role in their higher rates of obesity (CDC 2019).

The major sources of added sugars in the US diet are sugar-sweetened beverages (soft drinks, energy drinks, sports drinks, sweetened coffee and tea, alcoholic beverages, and flavored waters), providing almost half of total added sugars, and snacks and sweets (grain-based desserts, dairy desserts like ice cream, candy, jams, syrups, etc.), providing close to one-third of added sugars. The current Dietary Guidelines for Americans recommends a shift in eating patterns to reduce added sugar intake to less than $10 \%$ of calories (USDA 2015).

\section{Sugars and Nutrition}

You may wonder if raw sugar, honey, or agave nectar are more nutritious than other sugars. The answer is no. All of the following sweeteners are nutritionally the same; they contain calories along with no (or minimal) nutrients other than sugar (Duyff 2012).

Agave nectar: a nutritive sweetener produced from the heart of the agave plant. The fresh juice is heated, converting the complex carbohydrates into simple sugars, mainly fructose and glucose.

Raw sugar: coarse, granulated crystals formed from the evaporation of sugarcane juice. Raw sugar contains impurities and is not sold in stores. When the impurities are removed along with most of the molasses, the sugar can be sold as turbinado sugar.

Molasses: dark-colored syrup that is a by-product of sucrose production. It contains up to $70 \%$ sucrose. Molasses provides very small amounts of calcium and iron.

Brown sugar: sucrose crystals covered with small but varying amounts of molasses. 
Invert sugar: mixture of glucose and fructose formed by chemically splitting sucrose. Invert sugar prevents crystallization of cane sugar in candy.

Confectioners' or powdered sugar: finely ground sucrose crystals mixed with a small amount of cornstarch.

Honey: a sweetener containing fructose, glucose, maltose, and sucrose. It is made by bees and contains only trace amounts of vitamins and minerals.

Corn syrup: a sweetener made from cornstarch and used in many commercially prepared foods.

High-fructose corn syrup (HFCS): a sweetener made from cornstarch. An enzyme process slightly increases the fructose content, thus making HFCS sweeter than regular corn syrup. The glucose and fructose content of HFCS is similar to that of sucrose (table sugar).

\section{Sugar and Your Health}

Many people believe that sugar causes a variety of diseases, but there is no good evidence that sugar consumption per se is a significant factor in the development of heart disease, diabetes, hypoglycemia (low blood sugar), or hyperactivity. However, because eating a large amount of sugar can add many calories to the diet, it can contribute to the development of obesity, which is a risk factor for several other health problems.

Depending on the amount of sugar consumed, the impact on total calories consumed will vary. Eating one pound of sugar per week would add over 1,800 calories per week, which would have a significant effect on calorie balance. For many people, cutting down on sugar intake is helpful in decreasing caloric intake. Along with decreasing consumption of foods high in fat and increasing exercise, reducing foods with added sugars can be a significant factor in controlling obesity.

Sugar has replaced some complex carbohydrates in the diets of many Americans. Because complex carbohydrates are associated with other nutrients needed for good health, we are encouraged to include foods like beans, peas, and whole grain breads, pastas, and cereals in our diets. In order to increase our intake of nutritious high-carbohydrate foods without an increase in total calories, we must decrease the amount of sugar we consume.

\section{Sugar and Dental Health}

Sugar is a direct factor in the development of tooth decay. The type of sugar eaten and the frequency of consumption determine the likelihood that dental caries (cavities) will result. Sticky foods high in sugar tend to stay in contact with the teeth longer, increasing the chance of decay. Also, the more often during the day that teeth are exposed to sugar, the more likely it is that dental caries will occur. Heredity also plays a role, with some people being more susceptible to tooth decay than others. In order to avoid dental caries, avoid eating sugary snacks, and brush and floss your teeth as soon as possible after eating.

\section{Sugar in Modified Diets}

Many people are concerned about their sugar intake because of medical conditions, such as diabetes, that may require them to modify their use of refined carbohydrates and sugars. Blood glucose levels are controlled in part by the action of insulin, a hormone produced by the pancreas. When the blood glucose level rises, as it does after eating, the pancreas is stimulated to produce insulin, which facilitates glucose uptake into the cells and a return to an appropriate blood glucose level. Diabetes is a disease in which there is an inadequate supply of insulin or the hormone does not work properly. In either case, persons with diabetes are unable to regulate their blood glucose levels, and they must manage their diabetes through lifestyle choices and use of medications. Balancing carbohydrate intake throughout the day is an important part of a healthful diet for people with diabetes.

Hypoglycemia is a term meaning low blood glucose. It can be a symptom of various diseases. If you are worried that you may be hypoglycemic, consult your physician. Most people have relatively low blood glucose at certain times during the day. For example, when we wake up in the morning, our blood glucose may be lower than normal. However, a nutritious breakfast supplies the carbohydrates needed to push the blood glucose level back to normal. When your blood glucose level is low, you may feel tired, weak, and hungry. To relieve these symptoms, eat foods containing some protein and fat along with a complex carbohydrate. Consuming simple sugars in foods such as candy or soft drinks causes a quick but short-lived elevation of blood glucose. This is not the healthiest way to satisfy your morning hunger pangs.

Note that when the blood glucose of a person with diabetes becomes dangerously low, it is a medical emergency. This requires a rapid increase in blood glucose that only simple 
sugars can provide. Individuals with diabetes, especially those who take insulin or an oral medication that can cause hypoglycemia, must always carry a form of glucose with them to treat low blood glucose.

\section{High-Intensity Sweeteners}

High-intensity sweeteners provide alternatives to sugars in a variety of foods and beverages. They are classified as nutritive or non-nutritive sweeteners (NNS), depending on whether they provide calories.

The one nutritive high-intensity sweetener that is currently on the market in the United States is aspartame, commonly known by its trade name, Nutrasweet ${ }^{\circledR}$. The NNS approved in this country are advantame, neotame, sucralose, stevia glycosides (from the stevia plant), Luo Han Guo or monk fruit extract, saccharin, and acesulfame potassium (acesulfame K). Other NNS that are not currently approved for use in the United States include cyclamate, alimate, whole leaf and crude stevia extract, neohesperidin, and thaumatin. One or more of these may someday be available for use in the United States (CDC 2016; Duyff 2012).

Aspartame is a widely used reduced-calorie sweetener approved for use in the United States. Although aspartame provides the same number of calories per gram as sugar, it is about 200 times sweeter than sucrose, so a very small amount is needed to sweeten foods. Its taste is similar to that of sucrose, and it does not have an aftertaste. Aspartame breaks down rapidly under high heat and is less stable in liquids than in solid foods. Thus, taste problems may result when beverages are stored for long periods at high temperatures (e.g., keeping a case of diet soda in your hot garage).

The Food and Drug Administration (FDA) approved aspartame in 1974, and then withdrew its approval when questions were raised about aspartame's safety. After a court hearing and a review of the toxicological studies that had been performed, aspartame was again approved as safe for use in October 1981. When the FDA approved aspartame's use in carbonated beverages in 1983, some scientists and consumer groups raised concerns about the safety of aspartame's metabolites, which include phenylalanine, aspartic acid, methanol (wood alcohol), and diketopiperazine (DKP). Except for DKP, these substances are found naturally in a variety of foods or are formed in foods during cooking or processing. DKP was thoroughly tested and no adverse effects were identified. In February 1984, the FDA asked the Centers for Disease Control and Prevention (CDC) in Atlanta to investigate consumer complaints related to aspartame consumption. The CDC concluded that some individuals may be unusually sensitive to aspartame-containing products, but that there was no evidence of serious widespread adverse health consequences related to the use of the sweetener. People with the inherited disease phenylketonuria (PKU) cannot metabolize phenylalanine, and their diets have to be controlled to avoid brain damage. Therefore, all products containing aspartame must be labeled with a warning to persons with PKU that the product contains phenylalanine.

Saccharin is an NNS that is about 200 times sweeter than sucrose. Until 1981, it was the only NNS available in the United States after the ban on cyclamates in 1970. Although saccharin has been on the market since the early 1900 s, it was under investigation by the FDA due to studies that indicated a link between saccharin consumption and bladder cancer in rats. As a result of the original study reported in the early 1970s, the FDA removed saccharin from its "Generally Recognized As Safe" (GRAS) (FDA 2017) list of food additives, and in 1977, the FDA proposed a ban on the use of saccharin. The public outcry that followed this proposal led the FDA to put a moratorium on the ban until additional research could be done. The moratorium was extended by Congress every two years until 1991. For years, foods and beverages that contained saccharin were required to have a warning label on the package. The Saccharin Notice Repeal Act eliminated this requirement in 1996. Saccharin is most familiar as the sweetener Sweet'N Low ${ }^{\oplus}$, but more recently it also has been sold under the names Sweet Twin $^{\circledast}$ and Necta Sweet ${ }^{\circledR}$.

Acesulfame K (acesulfame potassium) was approved for use in specific food and beverage categories by the FDA in July 1988, and as a general-purpose sweetener and flavor enhancer in foods in 2003. It is marketed as Sunette ${ }^{\oplus}$ and Sweet One ${ }^{\oplus}$. Acesulfame $\mathrm{K}$ is not changed in the body and provides no calories or potassium. Because it does not break down at high temperatures, this sweetener can be used in cooking and baking. It is about 200 times sweeter than sugar.

Sucralose is a popular high-intensity NNS because of the way it pours and measures like sugar for use in baking. Sucralose is not metabolized in the body, so it contributes no calories when consumed. In 1998, sucralose was approved as a tabletop sweetener; in 1999, it was further approved as a general-use sweetener. Sucralose is sold under the brand name Splenda ${ }^{\circledR}$. Before approval, the FDA reviewed more than 110 studies to ensure safety in consumption. Sucralose is found in beverages, frozen desserts, gelatins, chewing gum, baked goods, and many more foods. 
Advantame is the most recently approved high-intensity NNS, only receiving FDA approval in 2014, as a sweetener and general flavor enhancer in foods. Even though advantame itself is a derivative of aspartame, so little of this sweetener is used at one time that the FDA does not require a label warning about the presence of phenylalanine for persons with PKU. Advantame is 20,000 times sweeter than sucrose and is heat-stable. As of April 2020, advantame was not available for commercial sale under a brand name or in any food products in the United States (USDA n.d.).

Neotame is sold under the brand name Newtame ${ }^{\circledast}$. At 7,000 to 13,000 times sweeter than sugar, neotame was given FDA approval in 2002 as a high-intensity sweetener and a flavor enhancer in foods. It is partially absorbed by the small intestine and rapidly metabolized before being fully excreted from the body. Like advantame, neotame contains phenylalanine in such a small concentration that the actual amount released in the body is negligible. Neotame does not change structure in high heat, which makes this product appropriate for baking.

Stevia glycosides are high-intensity NNS made from the Stevia rebaudiana Bertoni plant, more commonly known as stevia. In 1998, the FDA approved stevia glycosides for use as a food additive that is generally recognized as safe (GRAS). Products on the GRAS list are food additives that have met a standard of safety set by the FDA with a "reasonable certainty of no harm" in use. GRAS substances undergo a premarket review, as do other food additives, but the evaluation is done by experts who examine the safety of the product with publicly available scientific data. For a food substance (such as saccharin) that was GRAS certified before 1958, its use is considered safe "through experience based on common use in food" (FDA 2019).

Currently, purified rebaudioside A and stevioside are approved under the GRAS certification. Whole stevia leaves are not approved as an NNS, but they may be sold as a dietary supplement. Stevia is shelf-stable in dry form and more stable than acesulfame $\mathrm{K}$ and aspartame in liquid form.

Luo Han Guo extract is another GRAS product approved for use by the FDA in 2009. It is the common name for the compound Siraitia grosvenorii or Swingle fruit extract. The sweetness level varies from 150 to 300 times sweeter than sugar depending on the glycosides used. A commonly recognized name for this product is monk fruit, which has been used for centuries in China. Luo Han Guo extract is known to have an aftertaste at high levels.
Cyclamate is an NNS that is about 30 times sweeter than sucrose. It was a popular sweetener in carbonated beverages in the United States in the 1960s, often used in combination with saccharin. Cyclamate was banned in the United States in 1970 because rat studies implicated it was a carcinogen (a cancer-causing agent). The validity of the data has been questioned since then, but cyclamate remains unavailable in this country. However, it currently is marketed in some 40 countries, including Canada. In 1984, FDA's Cancer Assessment Committee reported that the evidence available indicated that cyclamate is not carcinogenic. The National Research Council of the National Academy of Sciences stated in 1985 that cyclamate is not carcinogenic and indicated that further research is not necessary.

\section{Intake of Non-nutritive Sweeteners and Health}

Studies comparing health outcomes of healthy adults or children with lower NNS intake compared to higher NNS intake were evaluated in a recent review of studies (Toews et al. 2019). The limited number of small intervention studies in adults showed a small reduction of body mass index (a measure associated with body fatness) and fasting blood glucose in individuals consuming NNS, but not in individuals consuming sucrose. Although several of the studies with shorter duration did not find a significant reduction in the energy intake in adults receiving NNS compared to sugar (Reid, Hammersley, and Duffy 2010, Reid et al. 2013, Reid et al. 2007), a longer 10-week study showed a lower energy intake in those consuming NNS (Raben et al. 2001). This may be due to daily sugar intake being lower in those who consume NNS (Raben et al. 2001, Reid, Hammersley, and Duffy 2010, Reid et al. 2013).

In children, a smaller increase in body mass index was observed with NNS intake compared to sugar (Toews et al. 2019). NNS intake does not seem to improve most health outcomes, including blood insulin levels, insulin resistance, pancreatic function, oral health, cancer, cardiovascular disease, chronic kidney disease, asthma, allergies, mood, or brain health. However, of the few studies identified for each health outcome, most were of short duration, had few study participants, and had other limitations. More research is needed to determine if there are relationships between intake of NNS intake and health.

Of recent interest is the potential relationships between NNS intake, gut microbiota, and health. Given their highintensity sweetness, most NNS are consumed in very low quantities. They are absorbed in the small intestine and do 
not exert any direct effects on the gut microbiota, which is in greatest abundance in the colon (Ruiz-Ojeda et al. 2019).

\section{What Food Labels Reveal}

The Nutrition Facts label currently in use shows the total amount of sugar in a product, but it does not distinguish between natural and added sugars. The new food label, approved by the FDA in 2016, provides this important information. Some food packages already feature the new Nutrition Facts panel, while companies have until July 2021 to comply. For those food packages using the old Nutrition Facts panel, make a habit of reading the ingredient list, which lists specific sugars as ingredients. If one of the terms meaning sugar is listed first or second in the ingredient list, then you know that sugar is one of the main ingredients by weight (see the example ingredient list below). The -ose suffix indicates that the substance is a sugar. Keep in mind that not all sugars have a name ending in -ose.

Sugars are ingredients in all sweet foods, but they also are found in other foods, including meat and poultry coating mixes, non-dairy coffee creamers, and salad dressings, among many other prepared foods and ingredients. Nutritive sweeteners in foods may be listed on the label as corn sweetener or natural sweetener. A natural sweetener could be honey, molasses, or date sugar.

\section{Example: Breakfast Drink Ingredient List}

INGREDIENTS: Nonfat dry milk, sugar ${ }^{\star}$, cocoa, sweet dairy whey, corn syrup solids ${ }^{\star}$, calcium caseinate, lactose ${ }^{\star}$, isolated soy protein, sodium caseinate, lecithin, magnesium hydroxide, carrageenan, artificial vanilla flavor, sodium ascorbate, ferric orthophosphate, vitamin E acetate, vitamin A palmitate, niacinamide, copper gluconate, zinc oxide, calcium pantothenate, thiamine mononitrate, pyridoxine hydrochloride, folic acid.

${ }^{\star}$ Sugar source.

\section{Breaking the Sugar Habit}

Once a person develops an eating habit or a desire for a food, it may be difficult to change that desire. One way to reduce your sugar intake is to gradually develop a taste for foods that are free from or low in added sugars. For example, learn to enjoy the taste of unsweetened fresh fruits. Gradually decrease the amount of sugar you add to tea and coffee, and select packaged foods and beverages, such as breakfast cereals, salad dressings, and fruit juices, that contain less added sugar.
The same holds true for sugar added when preparing and cooking foods. If you stop adding sugar to coleslaw, applesauce, and cooked vegetables, you may find that you like their natural flavors. In time, you and your family may find you can eliminate most of the added sugar from your diet.

Here are a few suggestions to make you more aware of your sugar consumption habits and to help you break them:

- Become aware of the sweet foods you eat. For the next several days, write down what you eat and put a check mark next to sweet foods. Also notice when and why you eat these sweet foods. Do you eat sweets out of boredom? Aim to decrease consumption of sweet foods and select more nutrient-rich choices.

- Make a list of foods you can substitute for sweets. For example, rather than soft drinks, you might drink milk, $100 \%$ fruit juice, water, or unsweetened tea (iced, with lemon); instead of pie, cookies, or sweet rolls, try wholegrain bread, fruit, or low-fat cheese slices; and instead of candy, snack on fruit or vegetable wedges.

- Search your existing recipe files and cookbooks. Many recipes use very little sugar and others can easily be modified. You can decrease the amount of sugar by onethird without significantly affecting the taste or texture of the product in many recipes.

- Read labels to estimate sugar content. Besides sugar, look for the words dextrose, maltose, sucrose, fructose, honey, corn sweetener, and corn syrup-they all mean sugar. If these appear in the first three ingredients, the product has a high sugar content. Also, if several of these different ingredients appear elsewhere in the listing, realize that if you add them all up, sugar might turn out to be the main ingredient (see Table 3 ).

- Buy fewer foods with a high sugar content. If they aren't in the cupboards or refrigerator, they won't be eaten as often.

- Substitute wholesome foods for some of the high-sugar foods you normally eat. Make sure you include a variety of foods from the five food groups daily.

- Avoid poor substitutes. Don't replace the sugar with high-fat foods or alcoholic beverages.

\section{Good Taste with Less Sugar}

There are many ways to reduce the amount of sugar eaten without giving up the taste you like. Be creative and experiment. Here are 28 tasty ideas to get you started: 


\section{Beverages}

- Substitute $100 \%$ fruit juice mixed with club soda for soft drinks, fruit juice cocktails, punches, and other drinks containing large amounts of sugar.

- Try unsweetened tea with a twist of lemon or a sprig of fresh mint.

- Enjoy water, mineral water, or club soda with a slice of fresh lime or lemon.

- Reduce consumption of soft drinks containing sugar; instead, choose $100 \%$ fruit juices (in small amounts), unsweetened iced tea, buttermilk, and low-fat milk.

- Add a stick of cinnamon or an orange slice to unsweetened coffee.

\section{Milk and Dairy Products}

- Substitute plain yogurt for fruit-flavored yogurt and add your own fresh fruit and a dash of cinnamon or nutmeg for flavor.

- Choose plain milk over chocolate milk, which has more sugar.

\section{Breads, Cereals, and Baked Goods}

- Eat fewer cookies, pies, and cakes. When you do have them, substitute graham crackers for other types of cookies, fruit pies for other types of pies, and plain cake with fruit toppings for frosted cakes. Eat small servings.

- Add fresh fruit or raisins instead of sugar to plain, readyto-eat breakfast cereals.

- Make cakes without frosting or frost only the top.

- Use yeast breads instead of sweet bread for the holidays. There are a variety to choose from and they use less sugar than traditional holiday breads.

- Experiment with recipes. Gradually decrease by onequarter to one-third the amount of sugar called for in baked items until you obtain an acceptable product. Bring out the flavor of the sweeteners with vanilla, lemon, or almond extract.

- Use fresh fruit toppings or unsweetened applesauce for pancakes, waffles, and French toast instead of syrup or honey.

\section{Protein Foods and Main Dishes}

- Leave out the sugar in stir-fried dishes. It's doubtful you'll even notice the difference.

- Make your own breading and coating mixes for meat and poultry. Some commercially prepared ones contain more than $50 \%$ sugar.
- Grind your own peanut butter (many grocery stores have machines for this) or look for peanut butter without added sugar.

\section{Fruits}

- Select fresh fruits, fruits canned in their own juice, or fruits canned in "lite" or "extra lite" syrup rather than heavy syrup.

- Use fruits rather than sugar to sweeten foods. Pineapple, raisins, bananas, oranges, and unsweetened fruit juices can be used in some vegetable and main dishes, as well as in desserts.

- Enjoy fresh fruit in season as a staple in your diet.

\section{Vegetables}

- Don't add sugar to vegetables when cooking. For extra flavor, try herbs and spices, or in some cases, fruits and $100 \%$ fruit juices.

- Read labels on frozen vegetables packaged in sauces or specialty packs. Many of these contain added sugar. Read labels on canned vegetables as well.

\section{Snacks}

- Substitute popcorn, raw vegetables, and fresh fruits for candy and other sweet snacks. Make these healthy foods easy to find and eat. Prepare them ahead of time and store in the refrigerator in an airtight plastic container labeled "snack foods."

- Make your own dips, because commercially prepared ones often contain sugar. Use raw vegetables as dippers in place of snack crackers, which often contain sugar as well.

\section{Condiments}

- Try making your own salad dressing. Many commercial dressings, both bottled and packaged mixes, contain a large amount of sugar.

- Try cardamom, coriander, basil, nutmeg, cinnamon, and ginger for a light, sweet taste.

- Read labels on bottled sauces and packaged seasoning mixes. You can easily make a similar version at home without the added sugar.

\section{Desserts}

- Get in the habit of serving fresh or simply prepared fruits for dessert. A fruit compote in orange juice, ambrosia, broiled grapefruit, or banana with cinnamon make tasty treats.

- Limit the frequency of sweet foods eaten. Eating two cookies at dinner will be less harmful for your teeth than one eaten in the afternoon and the other in the evening. 
For more information, contact your local UF/IFAS Extension family and consumer sciences agent. Your county UF/ IFAS Extension office may be listed in the government pages of your phone book. You can also find your local UF/IFAS Extension office online at https://sfyl.ifas.ufl.edu/ find-your-local-office/.

\section{References}

Centers for Disease Control and Prevention (CDC). 2019. "Know Your Limit for Added Sugars." Accessed April 15, 2020. https://www.cdc.gov/nutrition/data-statistics/knowyour-limit-for-added-sugars.html

Duyff, R. L. 2012. American Dietetic Association Complete Food \& Nutrition Guide, 4 Edition. New York: Houghton Mifflin Harcourt Publishing Company. 55-87.

U.S. Food and Drug Administration (FDA). 2018. "Additional Information about High-Intensity Sweeteners Permitted for Use in Food in the United States." Accessed April 15, 2020. https://www.fda.gov/Food/IngredientsPackagingLabeling/FoodAdditivesIngredients/ucm397725.htm

U.S. Food and Drug Administration (FDA). 2019. "Generally Recognized as Safe (GRAS).” Accessed January 22, 2020. https://www.fda.gov/Food/IngredientsPackagingLabeling/GRAS/default.htm

Mahan, L. K., and S. Escott-Stump. 2008. Krause's Food \& Nutrition Therapy, 12 Edition. St. Louis, MO: Saunders Elsevier. 43.

Raben, A., A. C. Møller, T. H. Vasilaras, and A. Astrup. 2001. "A Randomized 10 Week Trial of Sucrose vs Artificial Sweeteners on Body Weight and Blood Pressure After 10 Weeks." Obesity Research 9:86s.

Reid, M., R. Hammersley, and M. Duffy. 2010. "Effects of Sucrose Drinks on Macronutrient Intake, Body Weight, and Mood State in Overweight Women over 4 Weeks." Appetite 55 (1): 130-136. https://doi.org/10.1016/j. appet.2010.05.001.

Reid, M., R. Hammersley, M. Duffy, and C. Ballantyne. 2013. "Effects on Obese Women of the Sugar Sucrose Added to the Diet over 28 D: A Quasi-Randomised, SingleBlind, Controlled Trial." British Journal of Nutrition 111 (3): 563-570. https://doi.org/10.1017/S0007114513002687.
Reid, M., R. Hammersley, A. J. Hill, and P. Skidmore. 2007. "Long-Term Dietary Compensation for Added Sugar: Effects of Supplementary Sucrose Drinks over A 4-Week Period." British Journal of Nutrition 97 (1): 193-203. https:// doi.org/10.1017/S0007114507252705.

Ruiz-Ojeda, F. J., J. Plaza-Diaz, M. J. Saez-Lara, and A. Gil. 2019. "Effects of Sweeteners on the Gut Microbiota: A Review of Experimental Studies and Clinical Trials." Advances in Nutrition 10 (suppl_1): S31-S48. https://doi. org/10.1093/advances/nmy037.

Toews, I., S. Lohner, D. Küllenberg de Gaudry, H. Sommer, and J. J. Meerpohl. 2019. "Association between Intake of Non-sugar Sweeteners and Health Outcomes: Systematic Review and Meta-analyses of Randomised and Nonrandomised Controlled Trials and Observational Studies." BMJ 364:k4718. https://doi.org/10.1136/bmj.k4718.

United States Department of Agriculture (USDA). 2015. “Dietary Guidelines for Americans 2015-2020." Accessed April 15, 2020. http://health.gov/dietaryguidelines/2015/ guidelines/.

United States Department of Agriculture (USDA). n.d. "FoodData Central" Accessed April 15, 2020. https://fdc. nal.usda.gov/index.html 